\title{
Quantitative spectral CT evaluation of kidney tumors with the stretched-exponential nonlinear regression analysis model
}

\author{
Xiaotong Yang ${ }^{1,2 \#}$, Wenge Sun ${ }^{1 \#}$, Desheng Huang ${ }^{3}$, Hui $\mathrm{Li}^{4}$, Yu Zhao ${ }^{1}$, Peiling $\mathrm{Li}^{1}$, Yi Liu ${ }^{1}$ \\ ${ }^{1}$ Department of Radiology, The First Hospital of China Medical University, Shenyang, China; ${ }^{2}$ Department of Radiology, Liaocheng People's \\ Hospital, Liaocheng, China; ${ }^{3}$ Department of Mathematics, School of Fundamental Sciences, China Medical University, Shenyang, China; \\ ${ }^{4}$ Department of Radiology, Shengjing Hospital of China Medical University, Shenyang, China
}

\#These authors contributed equally to this work.

Correspondence to: Prof. Peiling Li; Prof. Yi Liu. Department of Radiology, The First Hospital of China Medical University, Shenyang 110001, China. Email: lipeilingcmu@163.com; Liuyicmu@sina.cn.

Background: This study aimed to use the stretched-exponential nonlinear regression analysis model to explore the value of the energy spectral curve in the differential diagnosis of clear cell renal cell carcinoma (ccRCC), minimal fat renal angiomyolipoma (RAML), and hypovascular renal cell carcinoma.

Methods: Sixty-five cases with renal tumors were enrolled retrospectively who had undergone a preoperative multiphase spectral CT scan of the kidney in pre-enhance and double-phase enhanced scanning. The normalized iodine concentrations (NIC) of these lesions, normal renal cortex, and psoas major were measured and calculated. The spectral curves of these lesions and normal tissues were analyzed to calculate the stretched-exponential index $(\alpha)$ and $b$ value with the stretched-exponential nonlinear regression analysis model $\left(y=-b \cdot X^{\alpha}\right)$. The differences between $\alpha, b$ value, and NIC of these lesions and normal tissues in preenhance and two enhanced phases were compared using one-way ANOVA. The correlation between $\alpha, b$ value, and NIC was evaluated using the Pearson coefficient test, with significance assigned at the $5 \%$ level.

Results: There was no significant difference in $\alpha$ value between the groups in pre-enhance scanning. In the Cortical phase (CP), there were no significant differences in NIC and $\alpha$ value between minimal fat RAML and hypovascular renal cell carcinoma, or between ccRCC and the normal renal cortex. However, in the nephrographic phase (NP), a significant difference in $\alpha$ value was found between minimal fat RAML and hypovascular renal cell carcinoma, but no difference in NIC between them. In NP, there were significant differences in NIC and $\alpha$ values between ccRCC and the normal renal cortex. In CP and NP, there were significant differences between the psoas major and other groups in all parameters. For b value, in preenhance scanning, there was a significant difference between the psoas major and other groups, and between ccRCC and the normal renal cortex. There was no significant difference between other groups. After enhancement, in CP and NP, significant differences were observed between the psoas major and other groups in b value, but no significant differences were observed between all renal tumors and the normal renal cortex. A linear correlation was found between $\alpha$ values and NIC in CP (r=0.780, P=0.00) and NP ( $r=0.693$, $\mathrm{P}=0.00)$. The b values and NIC had a low correlation in CP, with no correlation in NP.

Conclusions: Quantitative spectral CT with the stretched-exponential nonlinear regression analysis model may enhance the differential diagnosis ability for renal tumors. Its clinical value remains to be further explored in other types of soft tissue lesions.

Keywords: Spectral curve; renal tumor; stretched-exponential analysis model

Submitted Apr 12, 2020. Accepted for publication Oct 04, 2020.

doi: 10.21037/qims-20-562

View this article at: http://dx.doi.org/10.21037/qims-20-562 


\section{Introduction}

Computed tomography (CT) has provided effective images for diagnosing, treatment, and prognosis of tumors. The subsequent development of dual-energy spectral CT (DESCT) has further increased the role of CT in the evaluation of tumors. Compared to conventional CT, the advantage of DESCT lies in its ability to obtain multiparameter imaging, single energy imaging, quantitative analysis, and energy spectrum analysis (1-4). Additionally, it generates material decomposition (MD) images (water-, iodine-, and calcium-based), which can differentiate tumors by their signal patterns. It is reported that DESCT can provide a high-quality images of lesions by a single energy image and is also able to analyze the spectral curve of the lesions $(5,6)$, including analysis of stone composition and homology of lesions. Previous researchers have scanned various artificial solutions with DESCT and have analyzed the spectral curves through relevant mathematical models to obtain parameters that reflect the spectral curve $(7,8)$. These studies demonstrated the advantage of equations of spectral curves derived from the analysis of mathematical models, which provide the optimum method of reflecting the characteristics of the spectral curve and also maximize the clinical significance of spectral imaging. However, these studies had several shortcomings. Firstly, they were not able to reflect the physiological and pathological features of the intravital tissue, due to the various artificial solutions which were used in the experiments. Secondly, compared with complex human body tissue, artificial solutions are single components, making their spectral curve configuration difficult to compare to a real human body. For example, the curve of the glucose solution used in the research above is an up-convex curve model, while the curve of the intravital tissue (except for fat tissue) is usually an up-concave curve model (8). Thirdly, the mathematical equation used in the above research is complicated with several parameters, making it difficult to analyze the physiological significance of each parameter (7).

In this study, the stretched-exponential analysis model was applied to analyze the spectral curves of different pathological kinds of renal tumors compared with normal renal cortex and psoas major. We explored the physiological and pathological meaning of each mathematical parameter obtained from the stretched-exponential analysis model.

\section{Methods}

\section{Patient selection}

In this institutional review study approved by the hospital board, a total of 65 patients were retrospectively enrolled (41 men, 24 women), who had undergone preoperative multiphase DESCT of the kidney and were pathologically proven to have either ccRCC grade II (34 cases), minimal fat RAML (11 cases), or hypovascular renal cell carcinoma (9 cases of papillary renal cell carcinoma, 4 cases of chromophobe renal cell carcinoma, 4 cases of invasive urothelial cancer, 3 cases of Xpll.2 translocation/TFE3 gene fusion-associated renal cell carcinoma). Twenty-three of the 65 cases were selected randomly, and the contralateral renal cortex and psoas major of the 23 cases were used as the control group.

\section{Dual-energy CT examination}

Dual-energy CT scans were performed with a multidetector CT scanner (High-definition Discovery CT750HD, GE Healthcare, USA). Pre-enhance and two-phase enhanced CT examinations were performed using a spectral scanning mode (Gemstone Spectral Imaging, GSI). The preenhance scanning and enhancement scanning mode used the following protocol: instantaneous switching between voltages of 80 and $140 \mathrm{kVp}$; auto-tube current, $600 \mathrm{~mA}$; speed, $0.6 \mathrm{~s} /$ circle; display field of view (DFOV), $36 \mathrm{~cm}$; pitch, 0.984:1; layer thickness and interval, $5.0 \mathrm{~mm}$; layer thickness of reconstruction and interval, $1.25 \mathrm{~mm}$. Monochromatic images were obtained at values ranging from 40 to $140 \mathrm{keV}$ with an interval of $10 \mathrm{keV}$; the scan range was from the dome of the diaphragm to the superior anterior spine, including both sides of the kidneys. Patients were injected with non-ionic contrast medium iohexol (omnipaque, $350 \mathrm{mgI} / \mathrm{mL}$ ) via median cubital vein using a high-power injector (Medrad Stellant, USA) at a dose of $1.2 \mathrm{mg} / \mathrm{kg}$ for a total of $90 \mathrm{mg}$ at maximum. The iohexol must be injected in $25 \mathrm{~s}$. The delay after injection for CP imaging was determined using automatic image-triggering software (Smart Prep), and the ROI was set in the abdominal aorta at the level of the hepatic portal. When the trigger attenuation threshold (150 HU) was reached, the $\mathrm{CP}$ images were acquired. After a delay of $40 \mathrm{~s}$ after $\mathrm{CP}$, the NP images were acquired. 

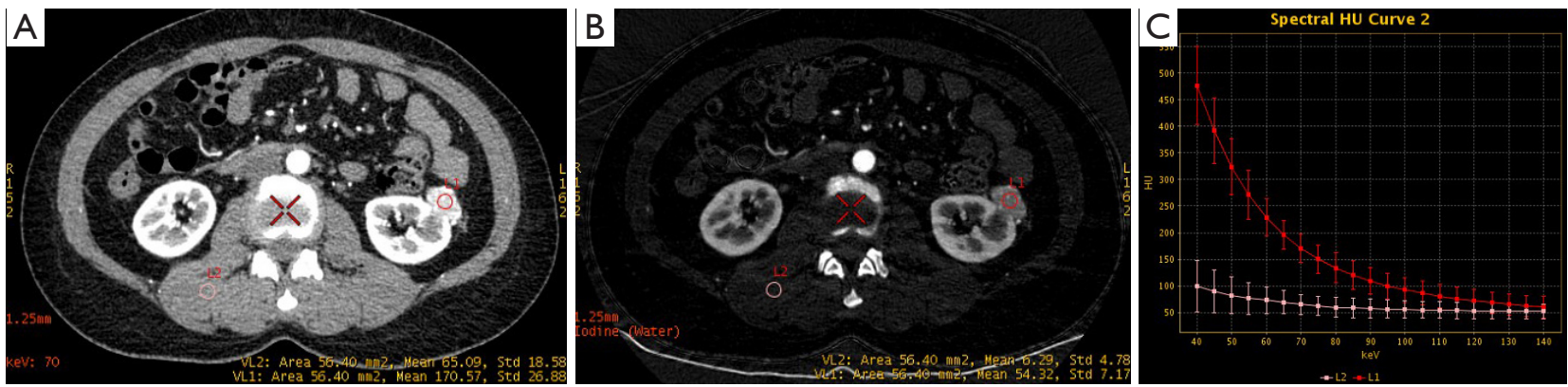

Figure 1 A 47-year-old woman with a mass in the left kidney pathologically confirmed as minimal fat RAML. (A) The monochromatic image at $70 \mathrm{keV}$ in the CP. (B) Iodine-based material decomposition image of this lesion at $70 \mathrm{keV}$ in the CP, the NIC of the lesion in CP is 0.388 . (C) Spectral curves of the tumor and psoas major. RAML, renal angiomyolipoma; CP, cortical phase; NIC, normalized iodine concentration.
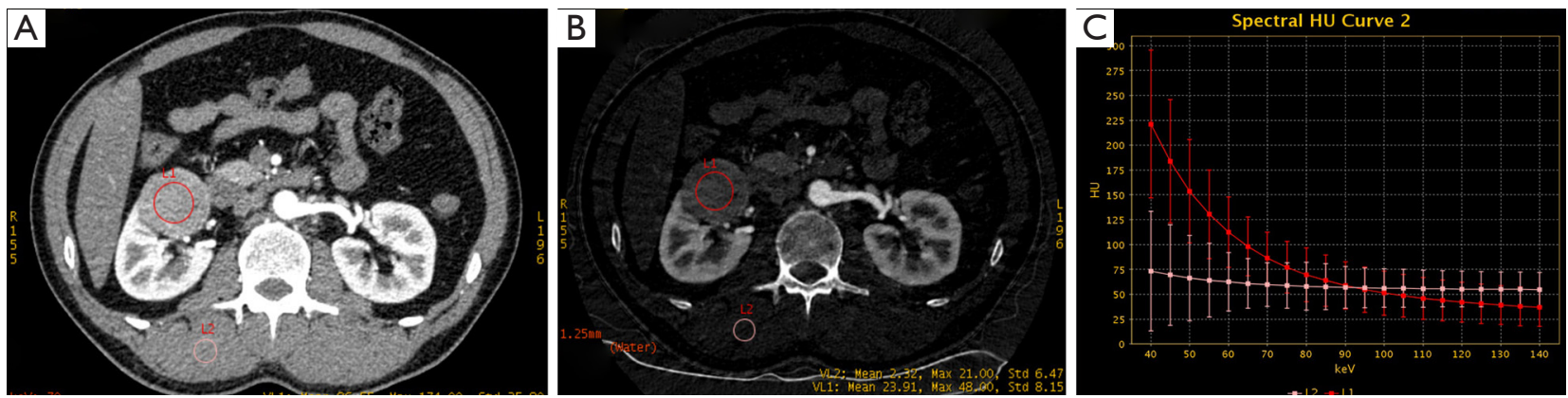

Figure 2 A 43-year-old man with a mass in the right kidney pathologically confirmed as chromophobe renal cell carcinoma. (A) The monochromatic image at $70 \mathrm{keV}$ in the CP. (B) Iodine-based material decomposition image of this lesion at $70 \mathrm{keV}$ in the CP, the NIC of the lesion in CP is 0.164. (C) Spectral curves of the tumor and psoas major. CP, cortical phase; NIC, normalized iodine concentration.

\section{Image analysis}

All the reconstructed images were measured on an advanced workstation (AW4.6) with the Gemstone Spectral Imaging (GSI) viewer. Regions of interest (ROIs) were placed over the lesions, normal renal cortex, aorta, and psoas major on the $70-\mathrm{keV}$ monochromatic images during the $\mathrm{CP}$ and NP, and large vessels, calcification, and areas of liquefaction necrosis were carefully avoided. Iodine concentrations (ICs) were derived from iodine-based material-decomposition CT images. To ensure consistency, all measurements were performed at the three adjacent continuous slices, and average values were calculated. For all measurements, the size, shape, and position of the ROIs were kept consistent between the two phases by applying the copy-and-paste function. The GSI viewer software package automatically obtains the spectral curve of the ROIs, which includes the CT values of the monochromatic images from 40 to $140 \mathrm{keV}$ (interval $10 \mathrm{keV}$ ) (Figures 1-3). Three parameters were derived from the spectral curve and IC measurements.
(I) To minimize bias of IC, the IC of tissues was normalized according to the IC of the aorta with the following formula.

$$
\mathrm{NIC}=\mathrm{IC}_{\text {tissue }} / \mathrm{IC}_{\text {aorta }}
$$

(II) The stretched-exponential index $(\alpha)$ and b values of the equation of the spectral curve.

$\mathrm{y}=-\mathrm{b} \cdot \mathrm{X}^{\alpha}, \mathrm{y}=\operatorname{Ln}\left(\mathrm{S} / \mathrm{S}_{0}\right)$, where $\mathrm{S}_{0}$ is the CT value in $40 \mathrm{keV}$, and $\mathrm{S}$ represents the CT values at different energy levels $(40-140 \mathrm{keV}$, interval $10 \mathrm{keV})$; $\mathrm{X}$ is the independent variable, defined as 11 consecutive integers from 0 to 10 .

\section{Statistical analysis}

The data were analyzed using SPSS version 16.0 (Chicago, IL, USA). Descriptive statistics (mean \pm standard deviation) were used to summarize all three quantitative variables. A one-way ANOVA was performed to test the NIC, $\alpha$, and $\mathrm{b}$ values (in $\mathrm{CP}$ and NP) among the five groups-namely, 

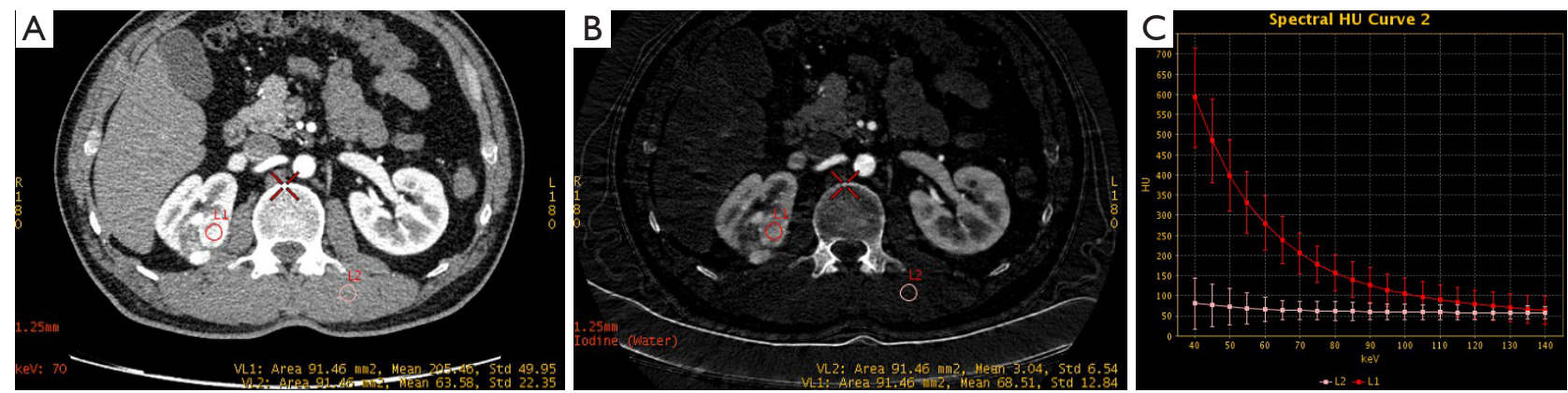

Figure 3 A 45-year-old man with a mass in the right kidney pathologically confirmed as ccRCC. (A) The monochromatic image at 70 keV in the CP. (B) Iodine-based material decomposition image of this lesion at $70 \mathrm{keV}$ in the CP, the NIC of the lesion in CP is 0.522 . (C) Spectral curves of the tumor and psoas major. ccRCC, clear cell renal cell carcinoma; $\mathrm{CP}$, cortical phase; NIC, normalized iodine concentration.

A

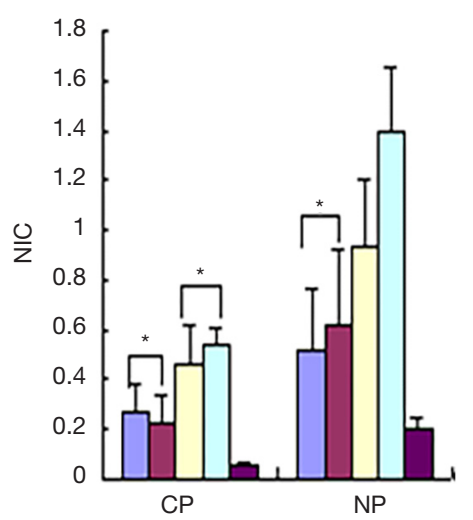

B

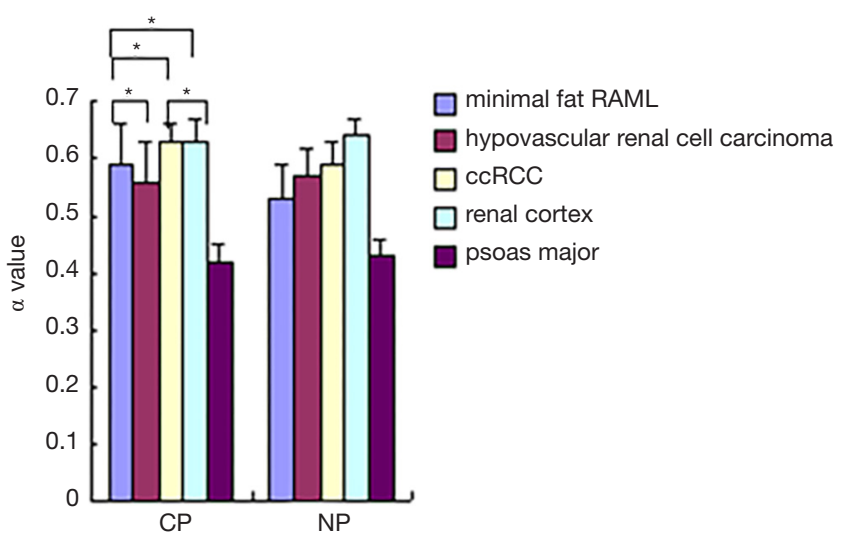

Figure $4 \alpha$ value (A) and normalized iodine concentration (NIC) (B) of the 5 kinds of organization structure in $\mathrm{CP}$ and NP. *, $\mathrm{P}>0.05$; the other groups $\mathrm{P}<0.05$. CP, cortical phase; NP, nephrographic phase.

minimal fat RAML, hypovascular renal cell carcinoma, ccRCC, the normal renal cortex, and the psoas major. Since it is difficult to differentiate between benign and malignant renal hypovascular tumors on radiographic images in the clinic, the receiving operating characteristics (ROC) curve was used to analyze these parameters of the benign and malignant renal hypovascular tumors (minimal fat RAML and hypovascular renal cell carcinoma). The Pearson correlation coefficient assessed the correlation between the value of $\alpha, b$, and NIC in CP and NP. P values less than 0.05 were considered statistically significant.

\section{Results}

Results indicated statistically significant differences in $\alpha$ and NIC values between some groups (Figure 4). There were no between-group differences in $\alpha$ values in pre-enhance scanning. In CP, there were no differences in NIC and $\alpha$ values between minimal fat RAML and hypovascular renal cell carcinoma, or between ccRCC and the normal renal cortex. However, in NP, there were statistically significant differences between minimal fat RAML and hypovascular renal cell carcinoma, but no differences in NIC in these two groups. In NP, there were statistically significant differences in NIC and $\alpha$ values between ccRCC and the normal renal cortex. In CP and NP, there were significant differences between the psoas major and other groups in all parameters. A positive correlation was found between $\alpha$ and NIC values amongst CP and NP (Figure 5A,B) (Tables 1,2).

In pre-enhance scanning, there were significant differences in $b$ values between the psoas major and the other groups, and between ccRCC and the normal renal cortex. However, there were no statistically significant differences in b values between other groups. After 

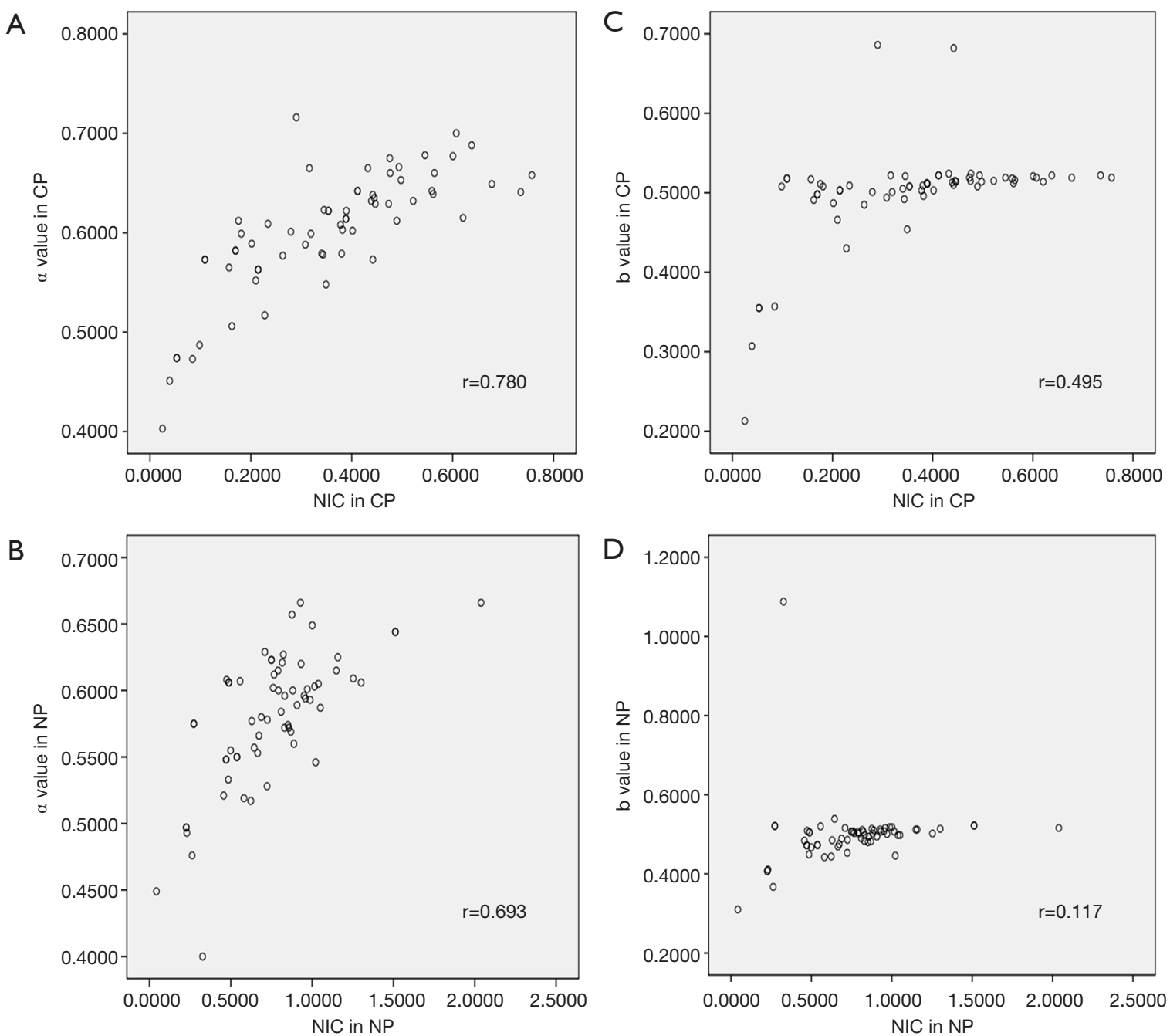

Figure 5 Scatter plots of correlations between $\alpha, b$ value, and NIC of these lesions. (A,B) Correlations between $\alpha$ value and NIC in the CP and NP ( $r=0.780, \mathrm{P}=0.000 ; \mathrm{r}=0.693, \mathrm{P}=0.000),(\mathrm{C}, \mathrm{D})$ Correlations between $\mathrm{b}$ value and NIC in the CP and NP $(\mathrm{r}=0.459, \mathrm{P}=0.000 ; \mathrm{r}=0.117$, $\mathrm{P}=0.353)$. NIC, normalized iodine concentration; $\mathrm{CP}$, cortical phase; NP, nephrographic phase.

enhancement, in CP and NP, there were significant differences in $b$ values between the psoas major and the other groups, but there were no significant differences in $\mathrm{b}$ values between all renal tumors and the normal renal cortex. A low positive correlation was found between $b$ values and NIC of these lesions in CP, but there was no correlation in NP (Figure 5C,D) (Tables 1,2).

To evaluate the diagnostic performance of NIC, $\alpha$, and $b$ values, the ROC curve was used to analyze these parameters of the benign and malignant renal hypovascular tumors (minimal fat RAML and hypovascular renal cell carcinoma). In CP, these three parameters could not differentiate the benign and malignant renal hypovascular tumors; the AUC was 0.473 (NIC), $0.473(\alpha)$, and $0.425(\mathrm{~b})$, respectively. In $\mathrm{NP}$, the $\alpha$ value had the highest AUC (0.709), followed by NIC (AUC 0.618), and b value (AUC 0.575) (Figure 6).

\section{Discussion}

DESCT, with its technology of rapid and periodical switching of tube voltages between 80 and $140 \mathrm{keV}$ in $0.5 \mathrm{~ms}$, has the advantage of being able to rapidly collect data while accurately obtaining the spectral decay curve and single-energy images of tissues, producing 110 singleenergy images on $40-140 \mathrm{keV}$ (9). The value of singleenergy CT imaging is that it is more accurate in evaluating 
Table 1 The actual estimates and interval estimates of $\alpha$ and $b$ values

\begin{tabular}{|c|c|c|c|c|c|c|}
\hline \multirow{3}{*}{ Groups } & \multicolumn{6}{|c|}{ 95\% confidence interval for mean (lower bound, upper bound) } \\
\hline & \multicolumn{2}{|c|}{ Pre-enhance scan } & \multicolumn{2}{|c|}{$\mathrm{CP}$} & \multicolumn{2}{|c|}{ NP } \\
\hline & $\alpha$ value & b value & $\alpha$ value & b value & $\alpha$ value & b value \\
\hline Group A $(n=11)$ & $0.37(0.345,0.383)$ & $0.22(0.176,0.271)$ & $0.58(0.534,0.624)$ & $0.49(0.434,0.553)$ & $0.53(0.499,0.569)$ & $0.53(0.398,0.654)$ \\
\hline Group B $(n=20)$ & $0.38(0.365,0.400)$ & $0.20(0.117,0.273)$ & $0.56(0.530,0.593)$ & $0.47(0.431,0.508)$ & $0.57(0.548,0.600)$ & $0.48(0.453,0.506)$ \\
\hline Group D $(n=23)$ & $0.37(0.334,0.404)$ & $0.32(0.262,0.353)$ & $0.63(0.613,0.649)$ & $0.52(0.513,0.533)$ & $0.64(0.630,0.654)$ & $0.52(0.514,0.520)$ \\
\hline Group E (n=23) & $0.36(0.337,0.385)$ & $0.10(0.071,0.128)$ & $0.42(0.399,0.446)$ & $0.28(0.241,0.322)$ & $0.43(0.413,0.452)$ & $0.30(0.264,0.344)$ \\
\hline
\end{tabular}

Table 2 The respective comparisons ( $\alpha, b$, and NIC values) between the tissues in the pre-enhance scanning, CP and NP

\begin{tabular}{|c|c|c|c|c|c|c|c|c|c|}
\hline Variable 1 & Variable 2 & \multicolumn{2}{|c|}{$\mathrm{NIC}$} & \multicolumn{3}{|c|}{$\alpha$} & \multicolumn{3}{|c|}{$b$} \\
\hline \multirow[t]{2}{*}{ Group A } & Group B & 0.917 & 0.381 & 0.774 & 0.845 & 0.015 & 0.544 & 0.894 & 0.924 \\
\hline & Group C & 0.008 & 0.000 & 0.979 & 0.414 & 0.000 & 0.253 & 0.995 & 0.961 \\
\hline \multirow[t]{3}{*}{ Group B } & Group C & 0.000 & 0.000 & 0.989 & 0.005 & 0.046 & 0.557 & 0.268 & 0.852 \\
\hline & Group D & 0.000 & 0.000 & 0.991 & 0.009 & 0.000 & 0.001 & 0.168 & 0.093 \\
\hline & Group E & 0.000 & 0.000 & 0.563 & 0.000 & 0.000 & 0.037 & 0.000 & 0.000 \\
\hline
\end{tabular}

Statistical methods: one-way ANOVA, P<0.05 was considered significant. Group A: minimal fat RAML; Group B: hypovascular renal cell carcinoma; Group C: ccRCC (grade II); Group D: renal cortex; Group E: psoas major. n, number of cases; CP, cortical phase; NP, nephrographic phase.

the absorption of X-rays in different materials than multienergy CT imaging; thus, we can obtain more accurate iodine concentrations (IC). Furthermore, it can calculate the decay parameters to find the subtle differences of various tissues by dealing with the spectral decay curve via mathematical equation. In this study, the stretchedexponential analysis model was used to analyze the spectral curves of different pathological types of renal tumors compared to the normal renal cortex and psoas major. We assessed the biological value of the stretched-exponential indices $(\alpha)$ and $b$ obtained from the stretched-exponential analysis.
The stretched-exponential index $(\alpha)$ demonstrated no statistical difference between normal tissues and lesions in pre-enhance scanning, indicating that the $\alpha$ index is not useful in distinguishing renal lesions from normal tissue in pre-enhance scanning. This may be because the density of renal lesions and normal tissue is similar, and thus the absorption of $\mathrm{X}$-rays is also similar and results in nil significant differences in $\alpha$ values. However, we found significant differences between the $\alpha$ values of normal tissue and renal lesion tissue in CP and NP, with a correlation between the $\alpha$ value and NIC. It is possible that the $\alpha$ value reflects the NIC in tissues due to the different density of 
A

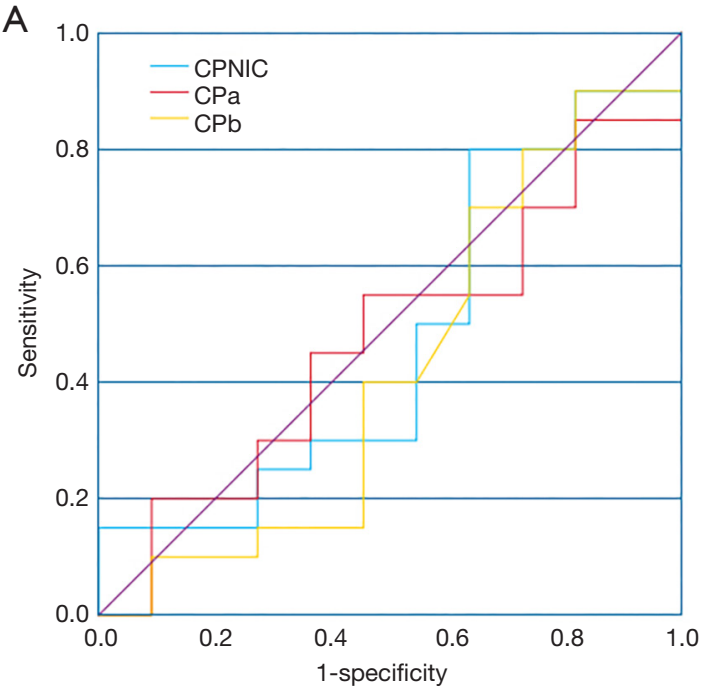

B

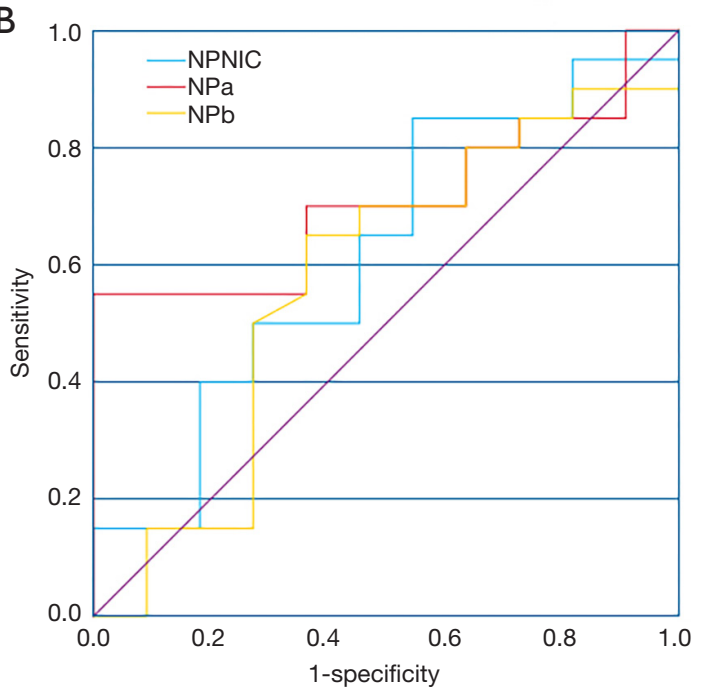

Figure 6 The receiving operating characteristics (ROC) curves of the benign and malignant renal hypovascular tumors in CP (A) and NP (B). CP, cortical phase; NP, nephrographic phase.

these issues, and which is reflected in the different ICs according to the various construction and circulation of microvessels contained therein. Other researchers have previously analyzed the spectral curves of contrast medium solutions with different IC via relevant mathematical equations (7). Their results suggest that spectral CT images could reflect the IC accurately, with some parameters positively linked to IC. Nguyen et al. revealed that the iodine base value is dependent on the materials separation mechanism-it is a highly accurate quantitative parameter. It could reflect the concentration of iodine in organs and tissues directly, thus reflecting the blood supply in organs and tissues indirectly (10). Moreover, the conclusion of previous research investigating MR intravoxel incoherent motion imaging has suggested that the value of $\alpha$, which is also calculated with the stretched-exponential analysis, is related to the complexity of the construction and composition of tissues (11). These outcomes were in agreement with our results.

There was no significant difference in the $\alpha$ value between minimal fat RAML and hypovascular renal cell carcinoma in CP. However, the $\alpha$ value of hypovascular renal cell carcinoma is higher than that of minimal fat RAML in NP $(\mathrm{P}<0.05)$. We suggest this result is due to the difference in the pathophysiologic character of benign and malignant renal tumors. As the $\mathrm{CP}$ in our study was acquired early (about $25 \mathrm{~s}$ after injection of the contrast agent), the contrast agent remained mainly in the artery, and thus the contrast agent concentration in the tissue would mostly be related to the arterial blood supply. Additionally, the number of intratumoral vessels which access the rapid artery blood supply are similar in both minimal fat RAML and hypovascular renal cell carcinoma, so the ICs in the two kinds of lesions are also likely to be similar.

For this reason, a comparison of the $\alpha$ value between ccRCC and the normal kidney cortex obtained similar results in $\mathrm{CP}$, whereas in NP, the intratumoral contrast agent concentration was related to intratumoral blood volume, extravascular volume, and capillary permeability. The malignant tumors have more neovascular processes and extravascular space, and higher capillary permeability than benign tumors and normal tissue, so the IC of hypovascular renal cell carcinoma should be higher than that of minimal fat RAML. Although the difference of NIC was not statistically significant in NP, it displayed a higher $\alpha$ value in hypovascular renal cell carcinoma than in minimal fat RAML. As the normal renal cortex has more microvessels than ccRCC, it is not unexpected that the IC of the normal renal cortex is higher than that of ccRCC. In $\mathrm{NP}$, this could account for the higher $\alpha$ value in the normal renal cortex than in ccRCC. These results indicated that the $\alpha$ value derived from spectral curve analysis could reflect the construction and microcirculation of soft tissues and, as such, this warrants further investigation.

In pre-enhance scanning, there were significant differences between the psoas major and other groups in $b$ 
value, and similarly between ccRCC and the normal renal cortex, but there were no statistically significant differences between the other groups. After enhancement, in CP and NP, there were statistically significant differences between the psoas major and other groups in b value, but there were no significant differences between all kinds of renal tumors and the normal renal cortex. This result suggests that the $b$ value may be related to the composition of soft tissues. The composition of psoas major is a muscle and is therefore of a different composition to renal tumors and the normal renal cortex, so the b value of psoas major is significantly different from that of these issues regardless of enhancement. In pre-enhance scanning, the $b$ value of ccRCC is lower than that of the normal renal cortex; however, the $\mathrm{b}$ values of minimal fat RAML and hypovascular renal cell carcinoma were similar, and the values lay between those of ccRCC and the normal renal cortex. On the one hand, the major components of minimal fat RAML and hypovascular renal cell carcinoma are parenchymal cells, suggesting that the $\mathrm{b}$ values should be similar.

On the other hand, ccRCC is a malignant tumor which should have more intercellular space and neovascular processes in addition to the tumor cells, whereas the normal renal cortex is composed of glomerulus and Bowman's capsule. Thus, it could also be argued that the $\mathrm{b}$ values should be different. After enhancement, in CP and NP, there were no significant differences in $\mathrm{b}$ values between all renal tumors and the normal renal cortex. Also, there was only a low correlation between the b values and NIC in CP, with no correlation in NP. This result might suggest that the $\mathrm{b}$ value is not associated with the blood supply of soft tissue, but this will need further investigation.

This study has some limitations. Firstly, we only chose the psoas major and normal renal cortex as the comparison to analyze the spectral curve of renal tumors, which limits the present results. Secondly, the spectral curves were influenced by image noise caused by current, voltage, ROI. Therefore, further study is required to prove the advantages of mathematical analysis on the spectral curve. Thirdly, the sample size in this study was small, and therefore these results require further validation to support the clinical significance of this stretched-exponential analysis on the spectral curve.

In conclusion, our study concentrated on applying a stretched-exponential nonlinear regression analysis model in order to analyze and obtain more information about spectral curves and to explore the practical significance of this method in differentiating between minimal fat RAML, hypovascular renal cell carcinoma, and preliminary ccRCC. The results indicated that the stretched-exponential index $(\alpha)$ might be related to the construction of tissues in preenhance scanning and the microcirculation of soft tissues after enhancement. Also, the b value may be related to the composition of soft tissues in pre-enhance scanning but appears to have no relationship with the blood supply of soft tissues. Therefore, it would seem that analyzing spectral curves with a stretched-exponential nonlinear regression analysis model could assist in the diagnosis of tumors, but further exploration will be required.

\section{Acknowledgments}

Funding: This study was supported by grants from the Foundation of Science and Technology Department of Liaoning Province, China (2013225049) to YL.

\section{Footnote}

Conflicts of Interest: All authors have completed the ICMJE uniform disclosure form (available at http://dx.doi. org/10.21037/qims-20-562). The authors have no conflicts of interest to declare.

Ethical Statement: This study was approved by the Ethics Review Board of the First Hospital of China Medical University and was performed in accordance with its guidelines.

Open Access Statement: This is an Open Access article distributed in accordance with the Creative Commons Attribution-NonCommercial-NoDerivs 4.0 International License (CC BY-NC-ND 4.0), which permits the noncommercial replication and distribution of the article with the strict proviso that no changes or edits are made and the original work is properly cited (including links to both the formal publication through the relevant DOI and the license). See: https://creativecommons.org/licenses/by-nc-nd/4.0/.

\section{References}

1. Wei W, Yu Y, Lv W, Deng K, Yuan L, Zhao Y. Predictive value of dual-energy spectral computed tomographic imaging on the histological origin of carcinomas in the ampullary region. Abdom Imaging 2014;39:702-10.

2. Lv P, Lin XZ, Li J, Li W, Chen K. Differentiation of small hepatic hemangioma from small hepatocellular carcinoma: 
recently introduced spectral CT method. Radiology 2011;259:720-9.

3. Wang L, Liu B, Wu XW, Wang J, Zhou Y, Wang WQ, Zhu XH, Yu YQ, Li XH, Zhang S, Shen Y. Correlation between CT attenuation value and iodine concentration in vitro: discrepancy between gemstone spectral imaging on single-source dual-energy CT and traditional polychromatic X-ray imaging. J Med Imaging Radiat Oncol 2012;56:379-83.

4. Matsumoto K, Jinzaki M, Tanami Y, Ueno A, Yamada M, Kuribayashi S. Virtual monochromatic spectral imaging with fast kilovoltage switching: improved image quality as compared with that obtained with conventional $120-\mathrm{kVp}$ CT. Radiology 2011;259:257-62.

5. Wu XW, Liu B, Wang L, Wang J, Wang WQ, Zhou Y, Li XH, Zhang S, Chen K, Shen Y. Correlation between blood-based screening of spectral CT and hemoglobin and total protein. Chin J Interv Imaging Ther 2011;8:518-20.

6. Zhao LQ, He W, Che JH, Li JY, Sun X. Correlation between hepatic arterial iodine fraction and hepatic

Cite this article as: Yang X, Sun W, Huang D, Li H, Zhao Y, Li P, Liu Y. Quantitative spectral CT evaluation of kidney tumors with the stretched-exponential nonlinear regression analysis model. Quant Imaging Med Surg 2021;11(2):676-684. doi: 10.21037/qims-20-562 arterial index of rabbits. Chin J Med Imaging Technnol 2011;27:2381-4.

7. Du XY, Li QW, Wang Y. Preliminary study on the quantitative analysis of iodine with spectral curve in CT spectral imaging. Radiol Pract 2013;28:616-8.

8. Zhang Y, Xing LH, Wu YY, Qin W, Li FT, Zhang J. Feasibility study of discriminating different solutions using spectral CT imaging. J Pract Radiol 2014;30:838-40.

9. Srinivasan A, Hoeffner E, Ibrahim M, Shah GV, LaMarca F, Mukherji SK. Utility of dual-energy CT virtual keV monochromatic series for the assessment of spinal transpedicular hardware-bone interface. AJR Am J Roentgenol 2013;201:878-83.

10. Nguyen NC, Kaushik A, Wolverson MK, Osman MM. Is there a common SUV threshold in oncological FDG PET/ CT, at least for some common indications? A retrospective study. Acta Oncol 2011;50:670-7.

11. Zhang Z, Yuan Q, Zhou H, Zhao D, Li L, Gerberich JL, Mason RP. Assessment of tumor response to oxygen challenge using quantitative diffusion MRI in an animal model. J Magn Reson Imaging 2015;42:1450-7. 